ROSSROADS

\title{
Human Space Exploration The Next Fifty Years
}

David R. Williams and Matthew Turnock

ABSTRACT: Preparation for the fiftieth anniversary of human spaceflight in the spring of 2011 provides the space faring nations with an opportunity to reflect on The International Space Station is a unique platform for long duration life science research that will play a critical role in preparing for future human space exploration beyond low earth orbit. Some feel the future path back to the Moon and on to Mars may be delayed with the current commitment of the United States to support the development of human-rated commercial spacecraft. Others see this as a unique opportunity to leverage the capability of the private sector in expanding access to space exploration. This article provides an overview of the past achievements in human spaceflight and discusses future missions over the next fifty years and the role space medicine will play in extending the time-distance constant of human space

Keywords: Human spaceflight, space medicine, career astronauts, spaceflight participants, commercial spaceflight

The past five decades will stand for eternity as that period in history when humans became a space faring species. From the first flights of Yuri Gagarin and Alan Shepard in the spring of 1961 the Apollo 11 lunar landing in 1969, people throughout the world were mesmerized by the incredible progress of space exploration. The decade culminated with the achievement of President Kennedy's vision of human exploration of the lunar surface and safe return to Earth. Meeting this goal required the combination of sophisticated engineering, life scicommitted to making what appeared impossible, possible.

From a clinical perspective bioastronautics (1) and space medicine (2) (3) research demon strated that humans could survive in space, work space and perform complex scientific missions on the surface of another celestial body. After the ex"To whom correspondence should be addressed: Department of Surgery McMaster University
39 Chaltron Avenue East, Hamilton, ON. L8N 4A6 ponential growth in short duration flight objectives on the development of a long duration capability for human spaceflight. The vision of sending human arther in to the solar system was shared by many experts within the United States and Russia. For that vision to become reality, the acclimation of humans to space had to be studied over the course of months not days. The Russian Salyut series of space stations and the NASA Skylab (4) (5) (6) program that highighted the next decade of human humans to live and work in microgravity for long periods of time. Both programs provided fundamental data on space physiology (7) relevant to space medicine but they also demonstrated the need for extended long duration missions on board a new generation of space stations.

The scientific utilization of space stations as microgravity research platforms provided an additional technical challenge in developing the capability to bring payloads to and from low earth orbit. The Space Shuttle was designed to meet this unique requirement along with additional roles as an autonomous science platform and as a vehicle that could be used to launch and repair satellites. The need for onboard robotics as a critical enabling technology was identified and Canada was invited to design and produce a robotic arm for the Shuttle program. Referred to as the Canadarm, or "the arm" for short, this contribution to the Shuttle program led to the first selection of six Canadian astronauts in 1983, with the prospect of a series of three flights for Canadian scientist astronauts referred to by NASA as payload specialists. Twenty-five years ago, Mark Garneau became the first Canadian to Ay in space aboard the Space Shutle Challenger. A number of dedicated Candian expenments were select for the STS-410 mission, crealing an hand experience with microgravity research. These experiments were referred to with the acronym CANEX, a descriptor which was used for the remaining two flights of Canadian payload specialists that took place in 1992 .

By this time the concept of a partnership of the major space faring nations working together to create a world-class orbiting research platform had become a reality, and the newly emerging space station program led to the need for, and selection of a second group of Canadian astronauts. During a six-month selection process, the Canadian Space Agency used a complex set of selection criteria to hire four new astronauts that would train as mission specialists for long duration missions on board the space station. Roberta Bondar and her back-up Ken Money retired after participating in the International Microgravity Laboratory (IML-2) mission in January 1992, leaving Marc Garneau, Bob Thirsk, Bjarni Tryggvason and Steve Maclean to be joined by Chris Hadfield, Julie Payette, Mike McKay and Dave Willams for mission specialist training and potential assignment to shuttle filights or space stafor the Condion Canadian profile as a major space faring nation, now with an expanded team of 8 astronauts, two with mission experience, capable of leveraging the Canadian robotic and scientific expertise.

The initial design requirements for the propod space station included a health maintenance facility (HMF) (8) in recognition of the potential medical issues that could arise during long duration missions in low earth orbit. In addition to the HMF the proposed airlock design provided both a hypobaric capability necessary for suited astronauts to egress the station for spacewalks as well as a hyperbaric capability to treat potential episodes of decompression sickness (DCS) that could arise during space walk. The designated operating pressure of the space station was 1 atmosphere (14.7 p.s.i.), similar to that of the Space Shuttle, while the sut pressure of the extravehicular mobility unit (EMU) was 4.3 p.s.l. thereby introducing the risk of DCS while transitioning to the lower suit pressure. Despite the rigorous design requirements for on board healthcare, these facilities were not implemented in the construction of the International Space Station primarily due to cost constrain

Historically, during the Shuttle era, the clina aproach to prevention, diagnosis and treatment of illness and injury in space had a strong through medical selection criteria, regular medica screening, and the development of countermea sures to mitigate the many physiologic changes associated with exposure to microgravity. This approach evolved from the early work in the Mercury, Gemini and Apollo programs that was based primarily on a preventive strategy with rudimentary on orbit diagnostic and treatment capabilities based on the use of small medical kits with support from fligh surgeons in mission control. The Skylab program provided an excellent opportunity for biomedical research during long duration missions that helped further delineate the physiologic changes associated with exposure to microgravity. These results were used to develop the exercise, cardiovascular and neurovestibular countermeasures implemented in the early Shuttle program and led to a further series of life science experiments conducted on dedicated Shuttle research missions. These studles were concluded by the launch of the first element of the International Space Station (ISS) in the fall of 1998 and were published a year later as a comprehensive extended duration orbiter (EDOMP) (9) (10).

Canadian researchers participated in a number of colme to help understand the many physiologic changes associated with acclimation to microgravity and to evaluate potential preventive countermeasures. In addition, researchers at the Canadian Space Agency (CSA) worked in collaboration with experts in DCS at the Defence Research and Development Canada Centre in Toronto to participate as one of three NASA supported research sites to develop the new pre-breathe protocols for use in preparation for spacewalks from the Interna- 
tional Space Station. This led to widespread recognition among the international partners of Canadian expertise in life science and space medicine research, which has continued into the current phase of ISS utilization.

The first twenty missions to the ISS were made up of three international crew members living aboard for approximately six months. Last year the crew configuration was extended to the original design requirement to accommodate six crew members for long duration missions thereby extending the capability to utilize the station as a research platform. During crew rotation Shuttle missions the number of crew increases to up to 13 during docked operations. With increased utilization, the While the preventive appreach of significant illness and injury has been effective, some believe it is a matter of time before thective, medical emergency in space. In addition to the career astronauts and cosmonauts living and working in space, a new ceneration of space flight porticipants (SFPs), or space tourists, have been visiting the space station since 2001.

Extending the opportunity to visit the ISS to space tourists challenged the space medicine community to decide whether or not a similar approach to pre-flight medical screening would be used for the SFPs. While these individuals were paying millions of dollars for the privilege of visiting the ISS, a significant on board medical event could have a profound mission impact and it was decided that a pre-flight medical assessment was needed as a risk miligation strategy. Seven SFPs have visited the ISS the most recent being Guy Laliberte from Canada, and to date no significant medical events have been reported.

Space medicine can be defined as the area of medical practice that deals with the provision of healthcare in partial and microgravitational environments. The scope of care not only deals with and injury in space, it involves pre-flight mess selection and conditioning as well as post-flight rehabilitation. The expansion of commercial space operations to include SFPs and potentially career astronauts flying on commercial spacecraft in suborbital and orbital flights presents a number of potential issues to the space medicine community. The FAA has released a series of requirements for crew and SFPs on commercial spacecraft in support of the Commercial Space Launch Amendments Act of 2004. These requirements are similar in nature to those used in commercial aviation with the additional stipulation that prospective SFPs sign an informed consent prior to flight (CFR 14 Part 460) (NO STYE for: ]). The FAA does not have a mandate to regulate passenger health or preflight medical screening on the proposed commercial spaceprits. Guidelines for screening SFPs have been published by the international space medicine community (12), yet it is uncertain whether or not commercial operators will implement them. Career astronauts utilizing commercial spacecraft to access low earth orbit will likely be governed by prefligh screening and a quarantine process similar to curently used protocols, yet this also remains to be

Despite rigorous screening programs, the increased size of the las crew coupled with the cy of flights by SFPs suggests that the frequency of on-orbit medical events may increase. While the majority of these events include space adaptation syndrome, motion sickness, back pain, musculoskeletal problems and disrupted sleep, the potential for a more significant medical event exists. For this reason, there has been considerable interes and research in developing and testing innovative diagnostic and therapeutic modalities over the past decade that will continue throughout ISS utilization The current paradigm for provision of healthcare on the ISS is based on dedicated crew medical officers (CMOs) utilizing the resources of the Crew Health Care System (CHeCS) to prevent, diagnose and treat on orbit medical events. A number of integrated medical kits are available on the ISS to treat the common medical problems that arise in space as outlined in the Integrated Medical Group medical operations checklist for expedition fights. In some cases the CMO is a physician, bu for the most part, they are crew members that have recelved additional medical training in preparation for the mission. CMOs provide care under the dion voice/video private medical conferences. This approach has been extremely effective in manaing the medical events that have taken place in space.

Additional equipment exists on the ISS to augment the $\mathrm{CHeCS}$ capability. The Human Research Facility - 1 (HRF-1) is equipped with a space-adapted, rack-mounted version of the HDI5000 Ultrasound System (ATL/Philips, Bothwell, WA). This unit has been used for a number of research studies (13) (14) evaluating various diag- nostic ultrasound protocols but is also available for clinical diagnostic use as needed. The portability and ease of use of diagnostic ultrasound have led to its adoption as the diagnostic imaging modality of choice in space. With current technology, other diagnostic imaging (DI) technologies are not practical for use in space. Further research on the role of diagnostic ultrasound and the development of alternative DI technologies is as important as an exploration enabling capability for future missions beyond low earth orbit.

Current on-board laboratory investigations are limited compared to terrestrial medicine. A number of research projects focus on developing portable blood analyzers (http://www.nsbri.org/NewsPublicouthelease.epl? $=89$ ) that will be a valuable future in the interim, flight surgeons rely heavily upon a linical history and physical examination to formulate a differential and primary diagnosis.

Past research efforts have focused on understanding the physiologic acclimation to microgravity with the development of a series of preventive countermeasures leading to further work that will continue to improve this preventive strategy. However, there is a tremendous amount of clinical research that is needed. The pharmacokinetics of drug use in microgravity and partial gravitational environments is an important area of further research (15) (16) while the effects of spaceflight on the pathophysiology of disease are either the subject of speculation or largely unknown. Building on the known physiologic acclimation to space, the pathophysiology of different diseases in space may be predictable, but some ilinesses such as DCS may be fundamentally different in microgravity (17). For the most part, therapeutic interventions in space involve the administration of medications orally, intramuscularly or intravenously. Foley catheters have been inserted for isolated cases of urinary retention (18) and intravenous access has cal interventions. There are no reported cases of wound repair using sutures or wound cement in humans, but anectal reports following anima surgery suggest that successful wound healing takes place with the use of wound cement. While number of studies of more advanced medical and surgical interventions have been evaluated in parabolic flight, further research and documentation of microgravity techniques for common interventions is warranted.
The future of human spaceflight will likely include increased accessibility and utilization of low earth orbit for commercial ventures and continued use of the ISS, ultimately leading to a transition back to exploration missions potentially involving lunar return or missions to Mars. The need to further deelop mission-specific medical capability involves discussions of the balance between the need for "stand-and-fight" capability and the utilization of "load-and-go approach to returning to Earth for definitive medical care. Currently approaches to on orbit health care use both approaches with the combination of immediate clinical care combined with the potential for an urgent or emergent deorbit and landing for definitive medical care. As human travel farther into space, a medical abort to Earth transitions to continued flight to the destination. This raises a number of questions about defining The appropriate level of care the effect of longer signal transmission to Earth on crew autonomy and the role that new technologies will play in the delivery of healthcare during the different phases of the mission.

On the ISS, as the complexity of medica interventions increases, there is greater reliance on round expertise through the use of telemedicine, fundamental component of linking the flight surdelivery in space (19). Telemedicine is the use of information and communication technology in near real-time to support medical providers at a distance. Many attempts have been made to evaluate and validate various protocols in extreme and remote environments that replicate the resource deficient and psychologically demanding conditions of human spaceflight (20). This has included a range of procedures from telementor-guided ultrasound to remotely operated surgical robotics (21) (22). As human space exploration extends further out into pose sigr support of health care delivery in space.

Planning for future exploration class mis-

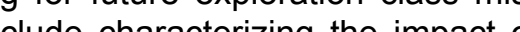
communication delay with existing telemedicine technology on real-time flight surgeon $\mathrm{CMO}$ coordination, defining the future skill sets and training re quirements for CMOs, as well as expanding the current capabilities to meet the future needs for space medical systems. For this reason, a comprehensive assessment of the likelihood and impact of potential medical conditions should be conducted based 
on a combination of historical data, expert opinion, analogue studies and epidemiological studies from other related high-risk occupations (23) to facilitate development of future medical protocols.

Unfortunately, the rarity and complexity of medical illness during spaceflight makes it difficut to evaluate the effectiveness of these protocols and new medical technologies. High-fidelity medical simulation has been suggested as an effective tool to assess the performance of high-level medical systems and interdependent medical teams (24). Electromechanical robotic mannequins can be used to simulate a wide variety of physiologic parameters, medical emergencies and illnesses in a controlled, reproducible, and isk-free environment to evaluate clinical protocols. Beyond research and for providing medical education and training opportunities for CMOs who may not be exposed to the required breadth of clinical experience necessary for supporting a space mission. In addition, it provides a context-specific opportunity for CMOs skill retention during a mission, or to provide just-in-time medical training to deal with an in-flight medical emergency.

The next decade provides an opportunity for further ISS research to develop new diagnostic and treatment capabilities, assess new technologies and evaluate strategies for $\mathrm{CMO}$ skill retention and just-in-time training. This research will be important to prepare for exploration class missions beyond low earth orbit in addition to developing on board clinical care for commercial space complexes. Based on the terrestrial approach of providing on-board healthcare for commercial ocean cruises, it is likely that commercial space complexes will have an on-board clinic with a physician or other health care professional providing clinical care. The evolution of commercial space travel in the decades to come will extend the scope of space medicine beyond the realm of the government supported flight whice the will differ between the government and commarial groups, there will be a shared interest amongst practitioners of pract approaches to prevent and treat illnesses and injuries during a mission. Clearly, the future apportunities for those interested in space medicine are very exciting.

\section{REFERENCES}

Per JF, West VR. Bioastronautics data book, second edition NASA-SP-3006. Parker JF, WestVR, editors. NASA Scientific and Technical Information Office; 1973a.

Link MM. Space medicine in project mercury NASASP-4003. Link MM, editors. Washington, DC: NASA Scientific and Technical Information Office; $1965 \mathrm{~b}$.

Johnston RS, Dietlein LA, Berry CA. Biomedical Results of Apollo. Johnston RS, Dietlein LA, Berry CA, editors. Washingon, DC. NASA Scientific and Technical Information Ofice; $1975 \mathrm{c}$.

The proceedings of the skylab life sciences symposium, Volume 1, 27-29 Aug. 1974, Houston, TX, United States 1974.

The proceedings of the skylab life sciences symposium Volume 2, 27-29 Aug. 1974, Houston, TX, United States

Michel EL, Johnston RS, Dietlein LF. Biomedical results of Pitts JA. The human factor: Biomedicine in the man space program to 1980 NASA-SP-4213 Washington. DC NASA Scientific and Technical Information Office; 1905

Billica RD, Doarn CR. A health maintence facility for space station freedom. Cutis 1991, Oct:48(4):315-8.

Sawin CF. Biomedical investigations conducted in support of the extended duration orbiter medical project. Avia Space Environ Med 1999, Feb;70(2):169-80

10. Sawin CF, Taylor GR, Smith WL. Extended Duration Orbiter Medical Project Final Report - NASA/SP-1999-534. Sawi CF, Taylor GR, Smith WL, editors. Washington, DC: NAS Scientific and Technical Information Office; 1999

2. Jennings RT, Murphy DM, Ware DL, Aunon SM, Moon RE, Bogomolov VV, et al. Medical qualification of a commercia spaceflight participant: Not your average astronaut. Avia Space Environ Med 2006, May;77(5):475-84.

13. Dulchavsky, Scott A. MD, PhD; Schwarz, Karl L. MD; Kirkpatrick, Andrew W. MD; Billica, Roger D. MD; Williams, David R. MD; Diebel, Lawrence N. MD; Campbell, Mark R. MD; Sargysan, Ashot E. MD, and; Hamilton, Douglas R. MD, PhD. Prospective evaluation of thoracic ultrasoun in 50 - Issue 2 - Pp 201-205 2001, Feb 1:50(2):2015.

14. Sir. PA, Kirkpe AW, et DA, FAST a MACH 20: Clinis trosound aboard the internationat space station. J Trauma 2005, Jan;58(1):35-9

15. Levy G. Pharmacodynamic aspects of spaceflight. The Journal of Clinical Pharmacology 1991:31(10):956.

16. Klaus DM, Howard HN. Antibiotic efficacy and microbia virulence during space flight. Trends Biotechnol 2006, Mar;24(3):131-6.
17. Williams DR. The biomedical challenges of space flight. Annu Rev Med 2003;54:245-56.

18. Stepaniak PC, Ramchandani SR, Jones JA. Acute urinary retention among astronauts. Aviat Space Environ Med 2007, Apr;78(4 Suppl):A5-8.

19. Williams DR, Bashshur RL, Pool SL, Doarn CR, Merrell RC, Logan JS. A strategic vision for telemedicine and medical informatics in space flight. Telemed $J E$ Health 2000;6(4):441-8.

20. Doarn CR. Telemedicine in extreme environments: Analogs for space flight. Stud Health Technol Inform 2003;97:35-41.

21. Dulchavsky S, Otto C, Comtois J-M, Sargsyan A, Dulchavsky A, Rubinfeld I. The martian chronicles: Remotely guided diagnosis and treatment in the arctic circle. Surg

Dave R. Williams obtained a Bachelor of Science, Major in Biology in 1976, a Master of Science from the Physiology Department, and a Doctorate of Medicine and a Master of Surgery from the Faculty of Medicine, McGill University. He further completed a residency in family practice in the Faculty of Medicine, University of Ottawa in 1985, and he obtained a fellowship in emergency medicine from the Royal College of Physicians and Surgeons of Canada, following completion of a residency in emergency medicine at the University of Toronto in 1988. In June 1992 the Canadian Space Agency selected Williams as one of four successful candidates from a field of 5330 applicants to begin astronaut training and flew aboard STS-90 and STS-118. He retired from active astronaut status in 2008 .

Matt Turnock (BSc., MSc) is a second year medical student at the University of Toronto, having recently completed his Master's of Science degree in eHealth at McMaster University, and prior to that a Masters of Space Studies at the International Space University. His research experience includes areas in telemedicine technology, high-fidelity simulation, neural network modelling, balance and posture analysis, and pre-hospital care in aeromedical transport. For fun he directs the Wilderness Medicine Interest Group, serves as section editor for the University of Toronto Medical Journal, and is an avid Dragonboat paddler. 